\title{
Approximate solution for system of fractional non-linear dynamical marriage model using Bernstein polynomials
}

\author{
Mohamed M. Khader ${ }^{a, b, *}$, Rubayyi T. Alqahtanic \\ ${ }^{a}$ Department of Mathematics and Statistics, College of Science, Al-Imam Mohammad Ibn Saud Islamic University (IMSIU), Riyadh: \\ 11566, Saudi Arabia. \\ ${ }^{b}$ Department of Mathematics, Faculty of Science, Benha University, Benha, Egypt. \\ ${ }^{c}$ Department of Mathematics and Statistics, College of Science, Al-Imam Mohammad Ibn Saud Islamic University (IMSIU), Riyadh: \\ 11566, Saudi Arabia.
}

Communicated by C. Alaca

\begin{abstract}
This paper is devoted to present the approximate solutions with helping of an efficient numerical method for the nonlinear coupled system of dynamical marriage model in the fractional of Riemann-Liouville sense (FDMM). The proposed system describes the dynamics of love affair between a couple. The proposed method is dependent on the use of useful properties of the operational matrices of Bernstein polynomials. The operational matrices for the fractional integration in the Riemann-Liouville sense and the product are used to reduce FDMM to the solution of non-linear system of algebraic equations using Newton iteration method. Numerical simulation is given to show the validity and the accuracy of the proposed algorithm. We introduce a comparison with the obtained solution using Runge-Kutta method. (C)2017 all rights reserved.
\end{abstract}

Keywords: Fractional dynamical model of marriage, Riemann-Liouville fractional derivatives, operational matrix, Bernstein polynomials.

2010 MSC: 41A04, 65N20.

\section{Introduction}

Fractional differential equations (FDEs) have been focused in many studies due to their frequent appearance in various applications in fluid mechanics, biology, physics and engineering [18]. Consequently, considerable attention has been given to the solutions of FDEs and integral equations of physical interest. In last decades, fractional calculus has drawn a wide attention from many physicists and mathematicians, because of its interdisciplinary application and physical meaning [16]. Fractional calculus deals with the generalization of differentiation and integration of non-integer order. Most FDEs do not have exact solutions, so approximate and numerical techniques ([6-13, 22-24]) must be used.

\footnotetext{
*Corresponding author

Email addresses: mohamed.khader@fsc.bu.edu.eg (Mohamed M. Khader), rtalqahtani@imamu.edu.sa (Rubayyi T. Alqahtani)

doi:10.22436/jnsa.010.03.02
} 
In recent decades the study of interpersonal relationships has begun to be popular. Interpersonal relationships appear in many contexts, such as in family (marriage), kinship, acquaintance, work and clubs [3]. Mathematical modeling in interpersonal relationships is very important for capturing the dynamics of people. But there are few models in this area and models have been restricted to integer order differential equations. Marriage has been studied scientifically for the past sixty years, and we can draw some general conclusions that guide our modeling of marital interaction [5]. Researchers are motivated by trying to understand why some couples divorce, but others do not, and why, among those who remain married, some are happy and some are miserable with one another. There are high levels of divorce in today's world. In the United States the current estimate is that within a forty year span approximately $50 \%$ to $67 \%$ percent of firs marriages will end in divorce; the figure is $10 \%$ higher for second marriage [15]. Although the United States has the highest raw divorce over time exist worldwide. Since experiments in these areas are difficult to design and may be constrained by ethical considerations, mathematical models can play a vital role in studying the dynamics of marriage and behavioral features.

The earliest known linear model of marriage is Romeo and Juliet model [21]. Suppose that at any time $t$, we could measure Romeo's love or hate for Juliet, $R(t)$, and Juliet's love or hate for Romeo, $J(t)$. Positive values of these functions indicate love, and negative values indicate hate. A very simple assumption would be that the change in Romeo's love for Juliet is a fraction of his current love plus a fraction of her current love. Similarly, Juliet's love for Romeo will change by a fraction of her current love for Romeo and a fraction of Romeo's love for her. This assumption leads us to the model equations

$$
\frac{d R}{d t}=a R(t)+b J(t), \quad \frac{d J}{d t}=c R(t)+d J(t),
$$

where $a, b, c$, and $d$ are constants.

Bernstein polynomials (BPs) are widely used in numerical computation. One of the advantages of using Bernstein polynomials as a tool for expansion functions is the good representation of smooth functions by finite Bernstein expansion provided that the function $u(t)$ is infinitely differentiable. The Bernstein collocation method reduces the problem to a system of non-linear algebraic equations.

The main aim of the presented paper is concerned with the application of the Bernstein collocation method to introduce the numerical simulation of the system of coupled non-linear fractional dynamical model of marriage.

\section{Preliminaries and notations}

In this section, we present some necessary definitions and mathematical preliminaries of the fractional calculus theory and the Bernstein polynomials that will be required in the present paper.

\subsection{The fractional integral and derivative operators}

Definition 2.1. The Riemann-Liouville fractional integral operator $J_{a}^{\alpha}$ of order $\alpha$ is defined on $L_{1}[a, b]$ as

$$
J_{a}^{\alpha} f(t)=\frac{1}{\Gamma(\alpha)} \int_{a}^{t}(t-\xi)^{\alpha-1} f(\xi) d \xi, \quad \alpha>0, \quad a \leqslant t \leqslant b .
$$

Definition 2.2. The Riemann-Liouville fractional derivative operator $D_{a}^{\alpha}$ of order $\alpha(n-1<\alpha \leqslant n)$, $n \in \mathbb{N}$ is defined in the following form

$$
D_{a}^{\alpha} f(t)=\frac{1}{\Gamma(n-\alpha)} \frac{d^{n}}{d t^{n}} \int_{a}^{t}(t-\xi)^{n-\alpha-1} f(\xi) d \xi, \quad \alpha>0, \quad a \leqslant t \leqslant b .
$$

Definition 2.3. The Caputo fractional derivative operator ${ }^{c} D_{a}^{\alpha}$ of order $\alpha(n-1<\alpha \leqslant n), n \in \mathbb{N}$ is defined in the following form

$$
{ }^{c} D_{a}^{\alpha} f(t)=\frac{1}{\Gamma(n-\alpha)} \int_{a}^{t}(t-\xi)^{n-\alpha-1} f^{(n)}(\xi) d \xi, \quad \alpha>0, \quad a \leqslant t \leqslant b .
$$


For more details on fractional derivatives definitions and its properties see $[16,18]$.

Lemma 2.4. If $\alpha \geqslant 0, \mathrm{n}=\lceil\alpha\rceil$ and $\mathrm{a} \leqslant \mathrm{t} \leqslant \mathrm{b}$, then

$$
\begin{aligned}
{ }^{c} D_{a}^{\alpha} J_{a}^{\alpha} f(t) & =f(t), \\
J_{a}^{\alpha c} D_{a}^{\alpha} f(t) & =f(t)-\sum_{i=0}^{n-1} \frac{f^{(i)}(a)}{i !}(t-a)^{i}, \\
{ }^{c} D_{a}^{\alpha} f(t) & =D_{a}^{\alpha} f(t)-\sum_{i=0}^{n-1} \frac{f^{(i)}(a)}{\Gamma(i-\alpha+1)}(t-a)^{i} .
\end{aligned}
$$

\subsection{Bernstein polynomials and their properties}

Definition 2.5. The $n+1$ Bernstein polynomials of degree $n$ are defined on the interval $[0,1]$ as follows [4]

$$
B_{i, n}(x)=\left(\begin{array}{c}
n \\
i
\end{array}\right) x^{i}(1-x)^{n-i}, \quad i=0,1, \ldots, n,
$$

where $\left(\begin{array}{c}n \\ i\end{array}\right)$ is a binomial coefficient.

The first few Bernstein basis polynomials are

$$
\begin{aligned}
& \mathrm{B}_{0,0}(\mathrm{x})=1 \text {, } \\
& \mathrm{B}_{0,1}(x)=1-x, \quad \mathrm{~B}_{1,1}(x)=x, \\
& \mathrm{~B}_{0,2}(\mathrm{x})=(1-x)^{2}, \quad \mathrm{~B}_{1,2}(x)=2 x(1-x), \quad \mathrm{B}_{2,2}(x)=x^{2} \text {, } \\
& \mathrm{B}_{0,3}(x)=(1-x)^{3}, \quad \mathrm{~B}_{1,3}(x)=3 x(1-x)^{2}, \quad \mathrm{~B}_{2,3}(x)=3 x^{2}(1-x), \quad \mathrm{B}_{3,3}(x)=x^{3} .
\end{aligned}
$$

The Bernstein polynomials have the following properties:

1. $B_{k, n}(x)=0$, if $k<0$ or $k>n$;

2. $B_{k, n}(0)=\delta_{k, 0}$ and $B_{k, n}(1)=\delta_{k, n}$ where $\delta$ is the Kronecker delta function;

3. $B_{k, n}(x) \geqslant 0$, for $x \in[0,1]$;

4. $\sum_{k=0}^{n} B_{k, n}(x)=1$;

5. they satisfy symmetry $B_{k, n}(x)=B_{n-k, n}(1-x)$;

6. $B_{k, n}^{\prime}(x)=n\left(B_{k-1, n-1}(x)-B_{k, n-1}(x)\right)$;

7. $\int_{0}^{1} B_{k, n}(x) d x=\frac{1}{n+1}, \forall k=0,1, \ldots, n$.

Since the set $\left\{B_{i, m}(x)\right\}_{i=0}^{m}$ in Hilbert space $L_{2}[0,1]$ is a complete basis, so, we can write any polynomial $u(x)$ of degree $m$ in terms of linear combination of $\left\{B_{i, m}(x)\right\}_{i=0}^{m}$ as in the following form

$$
u(x) \simeq \sum_{k=0}^{m} c_{k} B_{k, m}(x) .
$$

We can write $\Psi_{m}(x)=\left[B_{0, m}(x), B_{1, m}(x), \ldots, B_{m, m}(x)\right]^{\top}=A B_{m}(x)$, where $A$ is an upper triangular matrix, $\boldsymbol{B}_{\mathrm{m}}(\mathrm{x})=\left[1, \chi, \chi^{2}, \ldots, \chi^{\mathrm{m}}\right]^{\top}$. For more details about the definition, properties and the convergence analysis of Bernstein polynomials see [2].

\section{Mathematical model}

In this paper, we study the behavior of the approximate solution of a fractional order system for the dynamics of love affair between a couple [17]. The system which describes this model is given in the following form

$$
\begin{aligned}
& D^{\alpha} u(t)=-\lambda_{1} u(t)+\mu_{1} v(t)\left(1-\epsilon v^{2}(t)\right)+v_{1}, \\
& D^{\alpha} v(t)=-\lambda_{2} v(t)+\mu_{2} u(t)\left(1-\epsilon u^{2}(t)\right)+v_{2}, \quad t \in(0, L),
\end{aligned}
$$

with the initial conditions 


$$
u(0)=u^{0}, \quad v(0)=v^{0},
$$

where $0<\alpha \leqslant 1, \lambda_{i}>0, \mu_{i}$, and $v_{i}(i=1,2)$ are real constants. These parameters are oblivion, reaction and attraction constants, respectively. In the equations above, we assume that feelings decay exponentially fast in the absence of partners. The parameters specify the romantic style of individuals 1 and 2.

Recently, several authors, for example $[3,5]$ have investigated the nonlinear FDMM and its special properties. For more details on the proposed model see [15, 17].

\section{BPs operational matrix of Riemann-Liouville fractional integration}

Theorem 4.1 ([2]). The Bernstein polynomials operational matrix $F_{\alpha}$ from order $(m+1) \times(m+1)$ for the Riemann-Liouville fractional integral is defined as follows

$$
\mathrm{J}_{\mathrm{a}}^{\alpha} \Psi_{\mathrm{m}}(x)=\frac{1}{\Gamma(\alpha)} \int_{\mathrm{a}}^{\mathrm{t}}(\mathrm{t}-\xi)^{\alpha-1} \Psi_{\mathrm{m}}(\xi) \mathrm{d} \xi \approx \mathrm{F}_{\alpha} \Psi_{\mathrm{m}}(\mathrm{x}) .
$$

Definition 4.2. We can define the dual matrix $Q_{(m+1) \times(m+1)}$ on the basis of Bernstein polynomials of $m$ th degree as follows

$$
\mathrm{Q}=\int_{0}^{1} \Psi(x)(\Psi(x))^{\top} \mathrm{d} x
$$

where

$$
(Q)_{i+1, j+1}=\int_{0}^{1} B_{i, m}(x) B_{j, m}(x) d x=\frac{(m !)^{2}(2 m-i-j) !(i+j) !}{(2 m+1)(2 m) !(m-i) !(m-j) ! i ! j !}, \quad i, j=0,1, \ldots, m .
$$

Lemma 4.3 ([14]). Let $\mathrm{L}_{2}[0,1]$ be a Hilbert space with the inner product $\langle f, g\rangle=\int_{0}^{1} f(x) g(x) d x$ and $u(x) \in$ $\mathrm{L}_{2}[0,1]$. Then, we can find the unique vector $\mathrm{c}=\left[\mathrm{c}_{0}, \mathrm{c}_{1}, \ldots, \mathrm{c}_{\mathrm{m}}\right]^{\top}$ such that $\mathrm{C}^{\mathrm{T}} \Psi_{\mathrm{m}}(\mathrm{x})$ is the best approximation of $\mathrm{u}(\mathrm{x})$ from space $\mathrm{S}_{\mathrm{m}}=\operatorname{Span}\left[\mathrm{B}_{0, \mathrm{~m}}(\mathrm{x}), \mathrm{B}_{1, \mathrm{~m}}(\mathrm{x}), \ldots, \mathrm{B}_{\mathrm{m}, \mathrm{m}}(\mathrm{x})\right]$. Moreover, one can get $\mathrm{Q}^{-1}\left\langle\mathrm{u}(\mathrm{x}), \Psi_{\mathrm{m}}(\mathrm{x})\right\rangle$ such that $\left\langle u(x), \Psi_{m}(x)\right\rangle=\left[\left\langle u, B_{0, m}(x)\right\rangle,\left\langle u, B_{1, m}(x)\right\rangle, \ldots,\left\langle u, B_{m, m}(x)\right\rangle\right]^{\top}$.

Definition 4.4. Let $u(x)$ be a continuous function on the interval $[0,1]$. Then we can approximate $u(x)$ in the following polynomial of degree $n$ in Bernstein form [2]

$$
\bar{B}_{n}(u)(x)=\sum_{i=0}^{n} u\left(\frac{i}{n}\right) B_{i, n}(x) .
$$

It can be shown that it is uniformly convergent on the interval $[0,1]$,

$$
\lim _{n \rightarrow \infty} \bar{B}_{n}(u)(x)=u(x) .
$$

Theorem 4.5. Given a function $\mathrm{u}(\mathrm{x}) \in \mathrm{C}[0,1]$ and any $\delta>0$, there exists an integer $\mathrm{N}$ such that [2]

$$
\left|u(x)-\bar{B}_{n}(u)(x)\right|<\delta, \quad \forall n>N, \quad x \in[0,1] .
$$

The Bernstein polynomials operational matrix are used for solving many class of fractional differential equations, they used to solve numerically the fractional heat-and wave-like equations [20] and the multiterm orders fractional differential equations [19] and others [1].

\section{Implementation of Bernstein polynomials operational matrix for solving FDMM}

In this section, we introduce a numerical algorithm using Bernstein polynomials operational matrix method for solving the system of coupled non-linear fractional dynamical model of marriage (3.1). The 
proposed technique will be applied as in the following steps:

1. We use the initial conditions (3.2) to reduce the given problem (3.1) to a problem with zero initial conditions. So, we define

$$
\mathrm{u}(\mathrm{t})=\hat{\mathrm{u}}(\mathrm{t})+\overline{\mathrm{u}}(\mathrm{t}), \quad v(\mathrm{t})=\hat{v}(\mathrm{t})+\bar{v}(\mathrm{t}),
$$

where $\hat{u}(t)$ and $\hat{v}(t)$ are some known functions that satisfied the initial conditions (3.2) and $\bar{u}(t)$ and $\bar{v}(t)$ are new unknown functions.

2. Substituting (5.1) in (3.1) and (3.2), we have an initial-value problem as follows

$$
\begin{aligned}
& D_{0}^{\alpha} \bar{u}(t)=-\lambda_{1} \bar{u}(t)+\mu_{1} \bar{v}(t)-\mu_{1} \epsilon\left(p_{1}(t) \bar{v}(t)+p_{2}(t) \bar{v}^{2}(t)+\bar{v}^{3}(t)\right)+p_{3}(t), \\
& D_{0}^{\alpha} \bar{v}(t)=-\lambda_{2} \bar{v}(t)+\mu_{2} \bar{u}(t)-\mu_{2} \epsilon\left(q_{1}(t) \bar{u}(t)+q_{2}(t) \bar{u}^{2}(t)+\bar{u}^{3}(t)\right)+q_{3}(t),
\end{aligned}
$$

where

$$
\begin{array}{lll}
\mathrm{p}_{1}(\mathrm{t})=3 \hat{v}^{2}(\mathrm{t}), & \mathrm{p}_{2}(\mathrm{t})=3 \hat{v}(\mathrm{t}), & \mathrm{p}_{3}(\mathrm{t})=-\lambda_{1} \hat{\mathrm{u}}(\mathrm{t})-\mu_{1} \hat{v}(\mathrm{t})-\mu_{1} \epsilon \hat{v}^{3}(\mathrm{t})+v_{1}, \\
\mathrm{q}_{1}(\mathrm{t})=3 \hat{u}^{2}(\mathrm{t}), & \mathrm{q}_{2}(\mathrm{t})=3 \hat{\mathrm{u}}(\mathrm{t}), & \mathrm{q}_{3}(\mathrm{t})=-\lambda_{2} \hat{v}(\mathrm{t})-\mu_{2} \hat{\mathrm{u}}(\mathrm{t})-\mu_{2} \epsilon \hat{u}^{3}(\mathrm{t})+v_{2},
\end{array}
$$

subject to the initial conditions

$$
\bar{u}(0)=0, \quad \bar{v}(0)=0 .
$$

3. Using (2.2) in Lemma 2.4 we can write

$$
{ }^{c} D_{0}^{\alpha} \bar{u}(t)=D_{0}^{\alpha} \bar{u}(t), \quad{ }^{c} D_{0}^{\alpha} \bar{v}(t)=D_{0}^{\alpha} \bar{v}(t) .
$$

4. Using Lemma 3.3 in [2], the inputs $p_{i}(t), q_{i}(t)(i=1,2,3), D_{0}^{\alpha} \bar{u}(t)$ and $D_{0}^{\alpha} \bar{v}(t)$ can be approximated as follows

$$
\begin{aligned}
\mathrm{D}_{0}^{\alpha} \overline{\mathrm{u}}(\mathrm{t}) & \approx \mathrm{C}_{1}^{\top} \Psi_{\mathrm{m}}(\mathrm{t}), \quad \mathrm{D}_{0}^{\alpha} \bar{v}(\mathrm{t}) \approx \mathrm{C}_{2}^{\top} \Psi_{\mathrm{m}}(\mathrm{t}), \\
\mathrm{p}_{\mathrm{i}}(\mathrm{t}) & \approx \mathrm{P}_{\mathrm{i}}^{\top} \Psi_{\mathrm{m}}(\mathrm{t}), \quad \mathrm{q}_{\mathrm{i}}(\mathrm{t}) \approx \mathrm{Q}_{\mathrm{i}}^{\top} \Psi_{\mathrm{m}}(\mathrm{t}),
\end{aligned}
$$

where $P_{i}$ and $Q_{i}(i=1,2,3)$ are known $(m+1) \times 1$ column vectors, and $C_{1}$ and $C_{2}$ are unknown $(m+1) \times 1$ column vectors.

5. From (2.1), (4.1), (5.3), (5.4), and (5.5), we have

$$
\begin{aligned}
\bar{u}(t) & =J_{0}^{\alpha c} D_{0}^{\alpha} \bar{u}(t)+\sum_{i=0}^{n-1} \frac{\bar{u}^{(i)}(0)}{i !} t^{i}=J_{0}^{\alpha c} D_{0}^{\alpha} \bar{u}(t)=J_{0}^{\alpha} D_{0}^{\alpha} \bar{u}(t) \\
& =J_{0}^{\alpha}\left(C_{1}^{\top} \Psi_{m}(t)\right) \approx C_{1}^{\top} J_{0}^{\alpha} \Psi_{m}(t) \approx C_{1}^{\top} F_{1 \alpha} \Psi_{m}(t)=C_{1 \alpha}^{\top} \Psi_{m}(t), \\
\bar{v}(t) & =J_{0}^{\alpha c} D_{0}^{\alpha} \bar{v}(t)+\sum_{i=0}^{n-1} \frac{\bar{v}^{(i)}(0)}{i !} t^{i}=J_{0}^{\alpha c} D_{0}^{\alpha} \bar{v}(t)=J_{0}^{\alpha} D_{0}^{\alpha} \bar{v}(t) \\
& =J_{0}^{\alpha}\left(C_{2}^{\top} \Psi_{m}(t)\right) \approx C_{2}^{\top} J_{0}^{\alpha} \Psi_{m}(t) \approx C_{2}^{\top} F_{2 \alpha} \Psi_{m}(t)=C_{2 \alpha}^{T} \Psi_{m}(t),
\end{aligned}
$$

where $C_{i \alpha}^{\top}=C_{i}^{\top} F_{i \alpha,}(i=1,2)$.

6. By substituting (5.5)-(5.7) into (5.2), we obtain

$$
\begin{aligned}
C_{1}^{\top} \Psi_{m}(t)= & -\lambda_{1} C_{1 \alpha}^{\top} \Psi_{m}(t)+\mu_{1} C_{2 \alpha}^{\top} \Psi_{m}(t)-\mu_{1} \epsilon\left(P_{1}^{\top} \Psi_{m}(t)\left(\Psi_{m}(t)\right)^{\top} C_{2 \alpha}\right. \\
& \left.+P_{2}^{\top} \Psi_{m}(t) C_{2 \alpha}^{\top} \Psi_{m}(t)\left(\Psi_{m}(t)\right)^{\top} C_{2 \alpha}+\left(C_{2 \alpha}^{\top} \Psi_{m}(t)\right)^{3}\right)+P_{3}^{\top} \Psi_{m}(t), \\
C_{2}^{\top} \Psi_{m}(t)= & -\lambda_{2} C_{2 \alpha}^{\top} \Psi_{m}(t)+\mu_{2} C_{1 \alpha}^{\top} \Psi_{m}(t)-\mu_{2} \epsilon\left(Q_{1}^{T} \Psi_{m}(t)\left(\Psi_{m}(t)\right)^{\top} C_{1 \alpha}\right. \\
& \left.+Q_{2}^{\top} \Psi_{m}(t) C_{1 \alpha}^{\top} \Psi_{m}(t)\left(\Psi_{m}(t)\right)^{\top} C_{1 \alpha}+\left(C_{1 \alpha}^{\top} \Psi_{m}(t)\right)^{3}\right)+Q_{3}^{\top} \Psi_{m}(t),
\end{aligned}
$$


7. Then, from Lemma 3.5 in [2] we have

$$
\begin{aligned}
C_{i \alpha}^{\top} \Psi_{m}(t)\left(\Psi_{m}(t)\right)^{\top} & =\left(\Psi_{m}(t)\right)^{\top} \hat{C}_{i \alpha}, \quad i=1,2, \\
P_{i}^{\top} \Psi_{m}(t)\left(\Psi_{m}(t)\right)^{\top} & =\left(\Psi_{m}(t)\right)^{\top} \hat{P}_{i}, \quad i=1,2,3, \\
Q_{i}^{\top} \Psi_{m}(t)\left(\Psi_{m}(t)\right)^{\top} & =\left(\Psi_{m}(t)\right)^{\top} \hat{Q}_{i}, \quad i=1,2,3 .
\end{aligned}
$$

Therefore we can reduce (5.8) and (5.9) by using (5.10)-(5.12) as the following non-linear system of algebraic equations

$$
\begin{aligned}
& C_{1}=-\lambda_{1} C_{1 \alpha}+\mu_{1} C_{2 \alpha}-\mu_{1} \epsilon\left[\hat{P_{1}} C_{2 \alpha}+\hat{P_{2}} \hat{C}_{2 \alpha} C_{2 \alpha}+\left(C_{2 \alpha}\right)^{3}\right]+\hat{P_{3}} \\
& C_{2}=-\lambda_{2} C_{2 \alpha}+\mu_{2} C_{1 \alpha}-\mu_{2} \epsilon\left[\hat{Q_{1} C_{1 \alpha}}+\hat{Q_{2}} \hat{C}_{1 \alpha} C_{1 \alpha}+\left(C_{1 \alpha}\right)^{3}\right]+\hat{Q_{3}} .
\end{aligned}
$$

8. By solving this system we can obtain the vectors $C_{1}$ and $C_{2}$. Then, we can get

$$
\mathrm{u}(\mathrm{t}) \approx \hat{\mathrm{u}}(\mathrm{t})+\mathrm{C}_{1}^{\top} \mathrm{F}_{1 \alpha} \Psi_{\mathrm{m}}(\mathrm{t}), \quad v(\mathrm{t}) \approx \hat{v}(\mathrm{t})+\mathrm{C}_{2}^{\top} \mathrm{F}_{2 \alpha} \Psi_{\mathrm{m}}(\mathrm{t}) .
$$

\section{Numerical simulation}

In this section, we implement the proposed method to solve the system of coupled non-linear fractional dynamical model of marriage (3.1) with different values of the constants $\lambda_{1}, \lambda_{2}, \mu_{1}, \mu_{2}, \epsilon, v_{1}$, and $v_{2}$, different values of the fractional derivative $\alpha$ and different initial values.

The obtained approximate solutions by means of the proposed method are shown in Figures 1-9, where in Figures 1-3, we presented the behavior of the approximate solution with different values of initial values $\left(u^{0}, v^{0}\right)=(2,4),\left(u^{0}, v^{0}\right)=(1,2)$, and $\left(u^{0}, v^{0}\right)=(0.5,1)$, respectively, and $m=5$ with final time $t=600$.
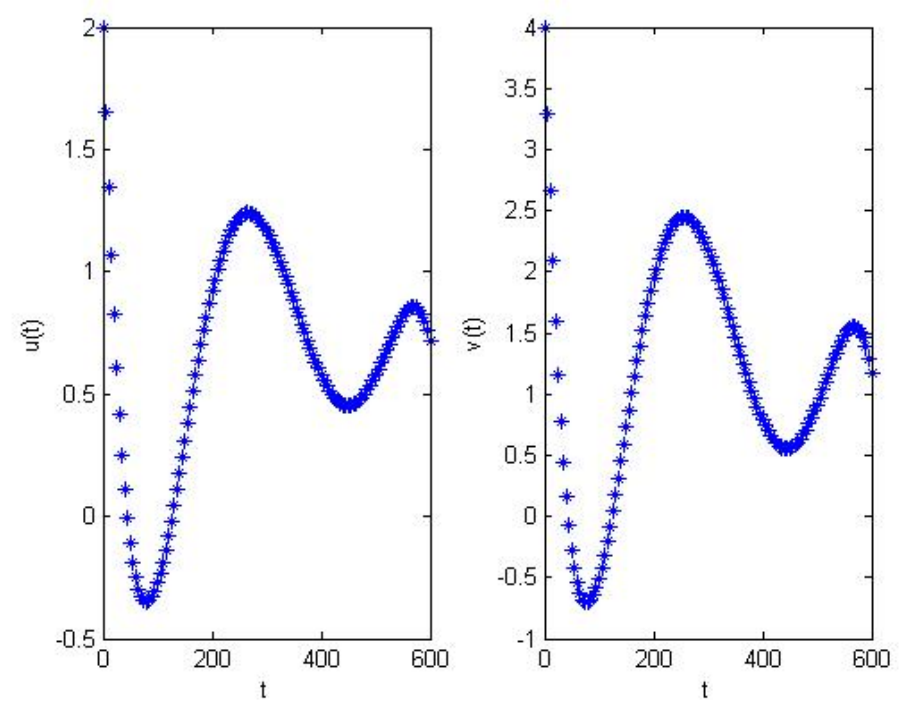

Figure 1: The behavior of the approximate solution with $\alpha=0.6, u^{0}=2, v^{0}=4$ and $\lambda_{1}=0.001, \lambda_{2}=0.004, \mu_{1}=0.005, \mu_{2}=$ $-0.001, \epsilon=0.01, v_{1}=0.02, v_{2}=0.03$.

In Figures 4-9, we presented the behavior of the approximate solution at $\alpha=0.9$ with different values of the other constants. From these figures, we can conclude that the obtained solution $u(t), v(t)$ confirms the natural behavior of the proposed system.

Also, in Figure 10, we presented the behavior of the approximate solution using the proposed method with the numerical solution obtained using fourth order Runge-Kutta method at $\alpha=1, u^{0}=v^{0}=0$ and $\lambda_{1}=0.003, \lambda_{2}=0.006, \mu_{1}=0.005, \mu_{2}=-0.001, \epsilon=0.01, v_{1}=0.05, v_{2}=0.05$. From this figure, we can conclude that this method gives results with an excellent agreement with the obtained solution $u(t), v(t)$ using Runge-Kutta method. 

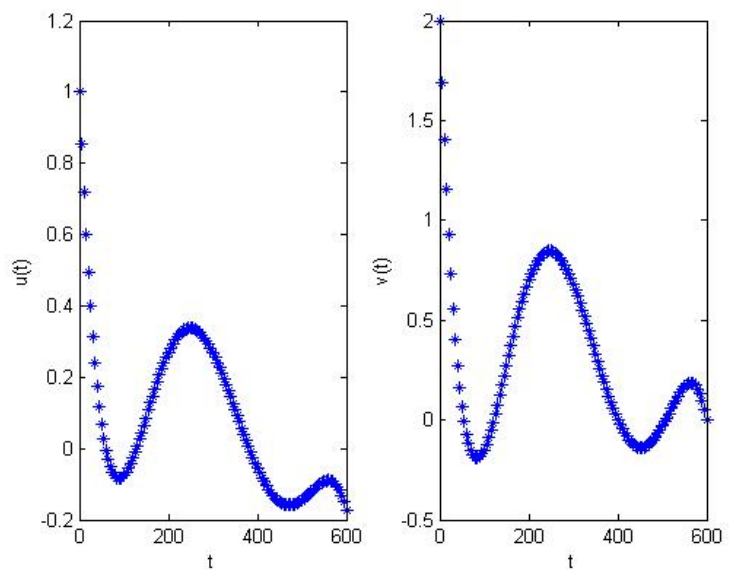

Figure 2: The behavior of the approximate solution with $\alpha=0.6, u^{0}=1, v^{0}=2$ and $\lambda_{1}=0.001, \lambda_{2}=0.004, \mu_{1}=$ $0.005, \mu_{2}=-0.001, \epsilon=0.01, v_{1}=0.02, v_{2}=0.03$.
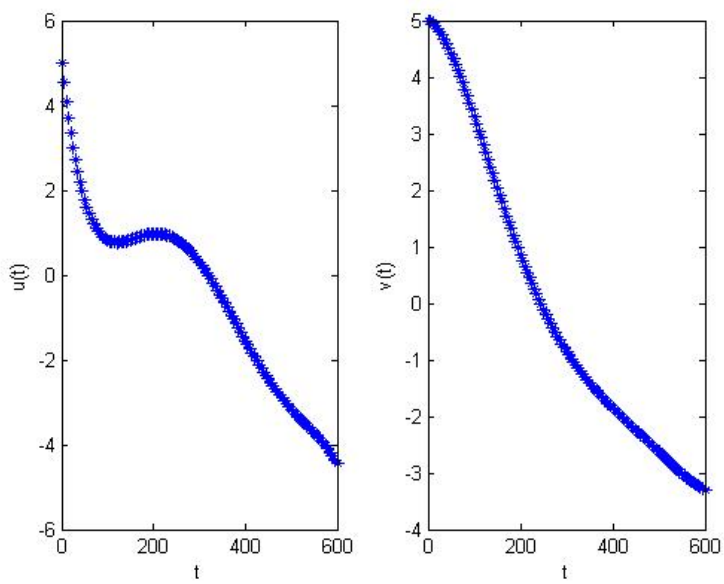

Figure 4: The behavior of the approximate solution with $\alpha=$ $0.9, u^{0}=v^{0}=5$ and $\lambda_{1}=0.001, \lambda_{2}=0.004, \mu_{1}=0.005, \mu_{2}=$ $-0.001, \epsilon=0.01, v_{1}=0.02, v_{2}=0.03$.
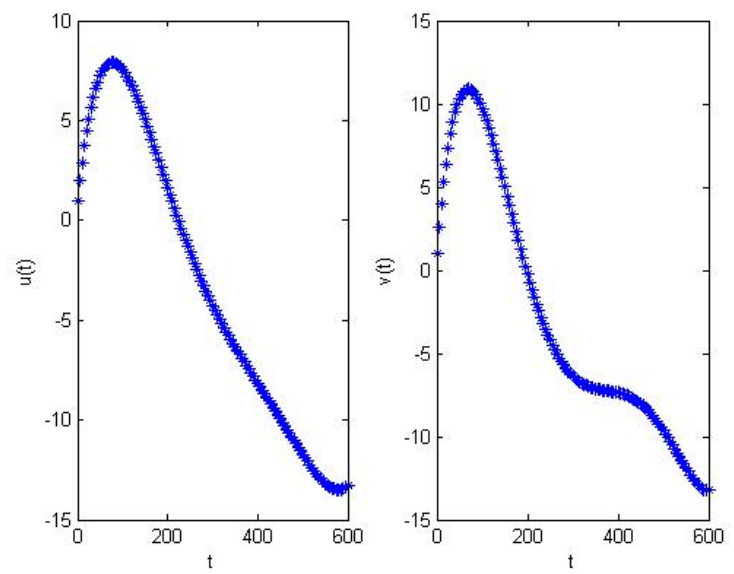

Figure 6: The behavior of the approximate solution with $\alpha=$ $0.9, \mathrm{u}^{0}=v^{0}=0$ and $\lambda_{1}=0.001, \lambda_{2}=0.004, \mu_{1}=0.005, \mu_{2}=$ $-0.001, \epsilon=0.01, v_{1}=0.07, v_{2}=0.09$.
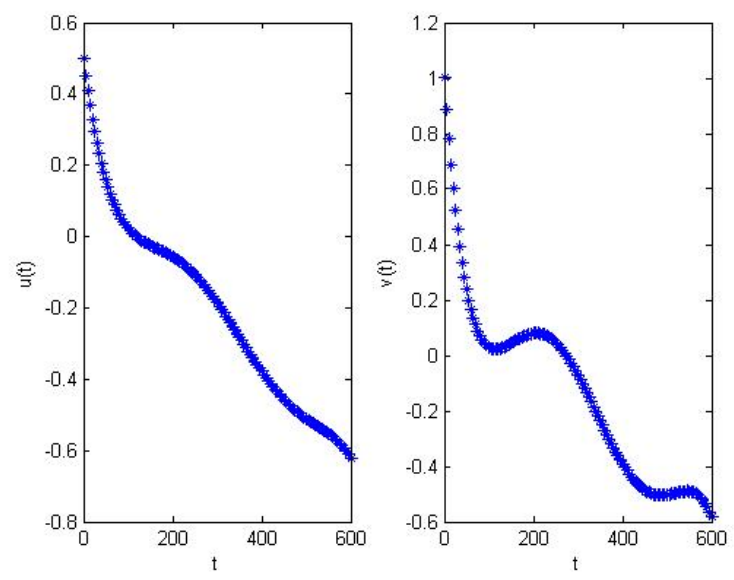

Figure 3: The behavior of the approximate solution with $\alpha=0.6, \mathrm{u}^{0}=0.5, v^{0}=1$ and $\lambda_{1}=0.001, \lambda_{2}=0.004, \mu_{1}=$ $0.005, \mu_{2}=-0.001, \epsilon=0.01, v_{1}=0.02, v_{2}=0.03$.
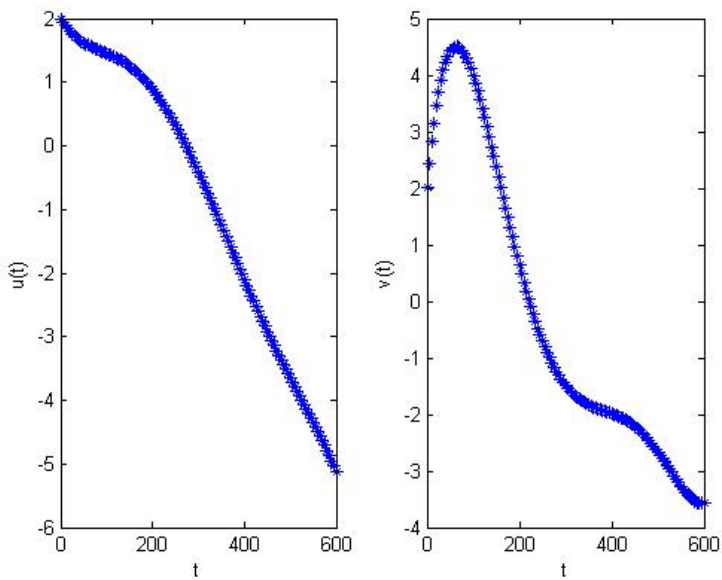

Figure 5: The behavior of the approximate solution with $\alpha=$ $0.9, u^{0}=v^{0}=2$ and $\lambda_{1}=0.001, \lambda_{2}=0.004, \mu_{1}=0.005, \mu_{2}=$ $-0.001, \epsilon=0.01, v_{1}=0.02, v_{2}=0.03$.
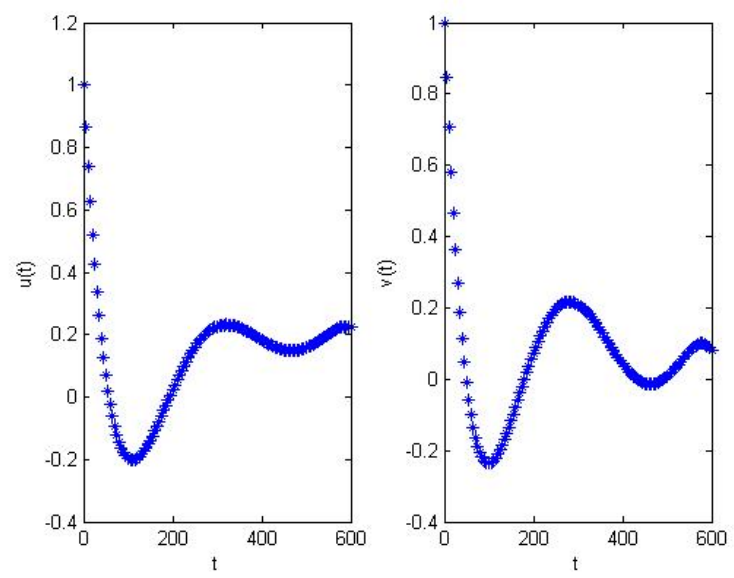

Figure 7: The behavior of the approximate solution with $\alpha=$ $0.9, u^{0}=v^{0}=1$ and $\lambda_{1}=0.001, \lambda_{2}=0.004, \mu_{1}=0.005, \mu_{2}=$ $-0.001, \epsilon=0.01, v_{1}=0.0, v_{2}=0.0$. 

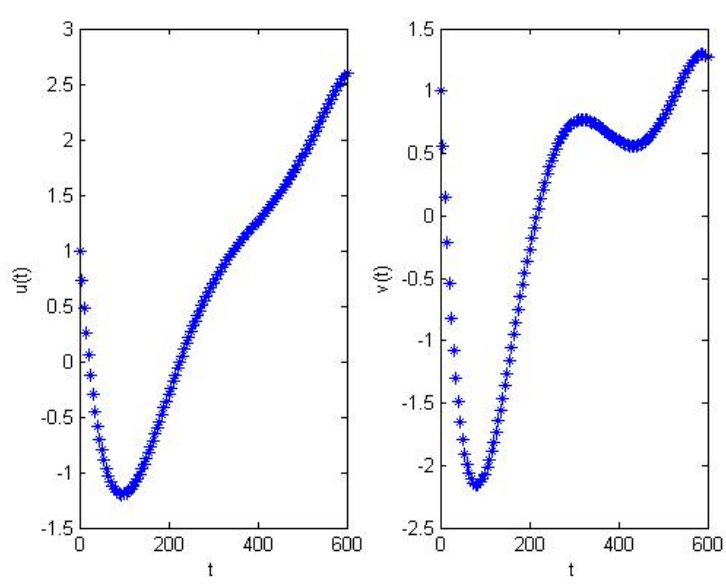

Figure 8: The behavior of the approximate solution with $\alpha=$ $0.9, u^{0}=v^{0}=1$ and $\lambda_{1}=0.001, \lambda_{2}=0.004, \mu_{1}=0.005, \mu_{2}=$ $-0.001, \epsilon=0.09, v_{1}=-0.01, v_{2}=-0.01$.
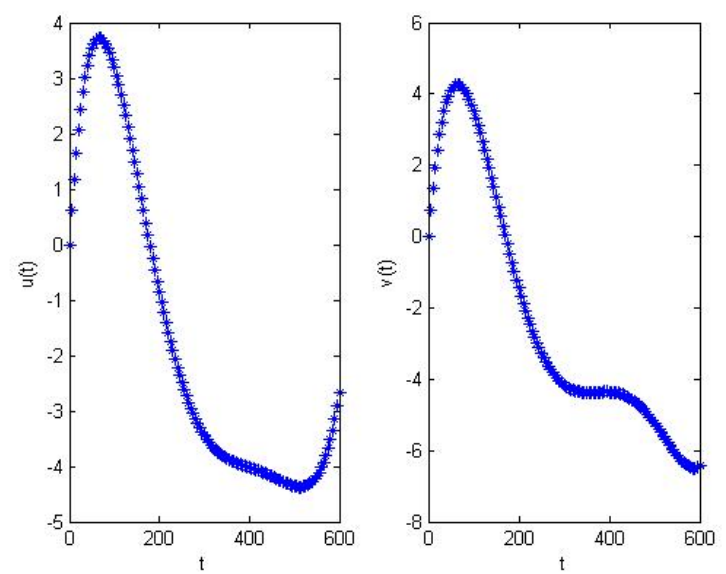

Figure 10: The behavior of the approximate solution and the numerical solution using RK4 at $\alpha=1$.

\section{Conclusion and remarks}

In this article, we used operational matrices of the Riemann-Liouville fractional integral and the product by Bernstein polynomials for solving the system of coupled non-linear fractional dynamical model of marriage. The fractional derivative is considered in the Riemann-Liouville sense. The properties of the Bernstein polynomials are used to reduce the proposed model to the solution of a system of nonlinear algebraic equations which is solved by using Newton iteration method. From the behavior of the obtained numerical solutions using the suggested method, we can see that the natural behavior of the proposed system is confirmed. Also, from the proposed comparison with the obtained solution using fourth order Runge-Kutta method (RK4), we can show that this approach can be solved the problem effectively. All computations in this paper are done using Matlab 8.

\section{References}

[1] M. Alipour, D. Rostamy, Bernstein polynomials for solving Abel's integral equation, J. Math. Comput. Sci., 3 (2011), 403-412. 4

[2] M. Alipour, D. Rostamy, D. Baleanu, Solving multi-dimensional fractional optimal control problems with inequality constraint by Bernstein polynomials operational matrices, J. Vib. Control, 19 (2012), 2523-2540. 2.2, 4.1, 4.4, 4.5, 4, 7 
[3] K. Barley, A. Cherif, Stochastic nonlinear dynamics of interpersonal and romantic relationships, Appl. Math. Comput., 217 (2011), 6273-6281. 1, 3

[4] E. W. Cheney, Introduction to approximation theory, Reprint of the second (1982) edition, AMS Chelsea Publishing, Providence, RI, (1998). 2.5

[5] J. M. Gottman, J. D. Murray, C. C. Swanson, R. Tyson, K. R. Swanson, The mathematics of marriage: dynamic nonlinear models, A Bradford Book, MIT Press, Cambridge, MA, (2002). 1, 3

[6] M. M. Khader, On the numerical solutions for the fractional diffusion equation, Commun. Nonlinear Sci. Numer. Simul., 16 (2011), 2535-2542. 1

[7] M. M. Khader, Numerical treatment for solving fractional Riccati differential equation, J. Egyptian Math. Soc., 21 (2013), 32-37.

[8] M. M. Khader, Numerical treatment for solving the perturbed fractional PDEs using hybrid techniques, J. Comput. Phys., 250 (2013), 565-573.

[9] M. M. Khader, M. H. Adel, Numerical solutions of fractional wave equations using an efficient class of FDM based on the Hermite formula, Adv. Difference Equ., 2016 (2016), 10 pages.

[10] M. M. Khader, T. S. EL Danaf, A. S. Hendy, A computational matrix method for solving systems of high order fractional differential equations, Appl. Math. Model., 37 (2013), 4035-4050.

[11] M. M. Khader, A. S. Hendy, A numerical technique for solving fractional variational problems, Math. Methods Appl. Sci., 36 (2013), 1281-1289.

[12] M. M. Khader, N. H. Sweilam, Singularly perturbed BVP to estimation of diaphragm deflection in MEMS capacitive microphone: an application of ADM, Appl. Math. Comput., 281 (2016), 214-222.

[13] M. M. Khader, N. H. Sweilam, A. M. S. Mahdy, Numerical study for the fractional differential equations generated by optimization problem using Chebyshev collocation method and FDM, Appl. Math. Inf. Sci., 7 (2013), 2011-2018. 1

[14] E. Kreyszig, Introductory functional analysis with applications, John Wiley \& Sons, New York-London-Sydney, (1978). 4.3

[15] T. C. Martin, L. L. Bumpass, Recent trends in marital disruption, Demography, 26 (1989), 37-51. 1, 3

[16] K. B. Oldham, J. Spanier, The fractional calculus, Theory and applications of differentiation and integration to arbitrary order, With an annotated chronological bibliography by Bertram Ross, Mathematics in Science and Engineering, Academic Press [A subsidiary of Harcourt Brace Jovanovich, Publishers], New York-London, (1974). $1,2.1$

[17] N. Ozalp, I. Koca, A fractional order nonlinear dynamical model of interpersonal relationships, Adv. Difference Equ., 2012 (2012), 7 pages. 3, 3

[18] I. Podlubny, Fractional differential equations, An introduction to fractional derivatives, fractional differential equations, to methods of their solution and some of their applications, Mathematics in Science and Engineering, Academic Press, Inc., San Diego, CA, (1999). 1, 2.1

[19] D. Rostamy, M. Alipour, H. Jafari, D. Baleanu, Solving multi-term orders fractional differential equations by operational matrices of BPs with convergence analysis, Rom. Rep. Phys., 65 (2013), 334-349. 4

[20] D. Rostamy, K. Karimi, Bernstein polynomials for solving fractional heat- and wave-like equations, Fract. Calc. Appl. Anal., 15 (2012), 556-571. 4

[21] S. H. Strogatz, Nonlinear dynamics and chaos: with applications to physics, biology, chemistry, and engineering, AddisonWesley, Reading, MA, (1994). 1

[22] N. H. Sweilam, M. M. Khader, A Chebyshev pseudo-spectral method for solving fractional-order integro-differential equations, ANZIAM J., 51 (2010), 464-475. 1

[23] N. H. Sweilam, M. M. Khader, A. M. S. Mahdy, Numerical studies for solving fractional-order Logistic equation, Int. J. Pure Appl. Math., 78 (2012), 1199-1210.

[24] N. H. Sweilam, M. M. Khader, A. M. Nagy, Numerical solution of two-sided space-fractional wave equation using finite difference method, J. Comput. Appl. Math., 235 (2011), 2832-2841. 1 